\title{
Adverse Effects, Adherence and Cost-Benefits in Glaucoma Treatment
}

\author{
Philippe Denis
}

University Professor, Hospital Consultant and Head, Ophthalmology Department, Croix-Rousse Hospital, Lyon

\begin{abstract}
Intraocular pressure (IOP)-lowering eye drops can prevent glaucoma progression and hence preserve vision, but adherence may be inadequate in as many as $59 \%$ of patients. Improving medication adherence is crucial for effective glaucoma control and represents a major challenge for physicians. Poor adherence, adverse events, poor health literacy and dissatisfaction with treatment are interrelated as well as being important risk factors for disease progression. Some adverse events may also reflect irreversible ocular damage with serious long-term consequences, including worsened outcomes following filtration surgery. Current treatment guidelines call for interventions that can maintain visual function and quality of life at a sustainable cost, including non-financial costs such as inconvenience and adverse events. Accumulating evidence suggests that the choice of first-line therapy is a leading determinant of long-term outcomes and economic costs. Effective early treatment that prevents or delays glaucoma progression could halve the long-term costs of disease management. Selecting an effective and well-tolerated IOP-lowering formulation is thus a vital first step to achieving effective disease control for the patient's lifetime. A holistic approach to patient management should comprise a straightforward regimen including effective and well-tolerated drugs (and excipients), combined with patient-centred communication to optimise disease understanding. Available data suggest that addressing all known causes of non-adherence from the start may prove to be the best strategy for managing glaucoma. Ongoing research offers potential drug delivery technologies that could increase patient adherence while improving efficacy and decreasing adverse effects and, ultimately, maintaining vision for glaucoma patients. Until these innovations are introduced, we can only choose the best options currently available.
\end{abstract}

\section{Keywords}

Glaucoma, compliance, benzalkonium chloride, preservative, intraocular pressure

Disclosure: The author has received consultant fees from Alcon, Allergan, MSD and Pfizer.

Acknowledgements: Writing support was provided by Touch Briefings.

Received: 10 October 2011 Accepted: 14 November 2011 Citation: European Ophthalmic Review, 2011;5(2):116-22 DOl: 10.17925/EOR.2011.05.02.116

Correspondence: Philippe Denis, Service d'Ophtalmologie, Hôpital de la Croix-Rousse, 103, Grande Rue de la Croix-Rousse, 69317 Lyon cédex 04, France.

E: philippe.denis@chu-lyon.fr

Support: The publication of this article was funded by Santen Oy. The views and opinions expressed are those of the author and not necessarily those of Santen Oy.

Controlling intraocular pressure (IOP) has proven efficacy in preventing glaucoma progression and remains central to disease management. ${ }^{1-3}$ Reducing IOP prevents or delays the onset of open-angle glaucoma in patients with ocular hypertension and slows progression among those with open-angle glaucoma.

Medication can preserve visual function long term, but poor adherence is a major issue affecting around $22-59 \%$ of patients. ${ }^{4-6}$ Barriers to adherence fall into four main categories:

- situational/environmental factors, such as travel or variation in routine;

- medication regimen, such as adverse effects or complexity;

- patient factors, such as skill in application and memory; and

- provider factors, such as communication and satisfaction with care.

Physicians can influence adherence strongly via two of these categories: medication regimen and provider factors.

There is a shift away from a goal of maximum efficacy in glaucoma management - that is, achieving the strongest IOP reduction possible - and towards optimal effectiveness - that is, sufficient IOP reduction to preserve visual function while minimising adverse effects, inconvenience and economic costs. ${ }^{8}$ This more pragmatic approach acknowledges the impact of adverse effects and adherence on efficacy and offers an opportunity to improve outcomes without increasing costs.

This article reviews the influence of factors such as adverse effects on adherence and how this affects efficacy and hence glaucoma progression, before discussing how clinicians can apply their growing understanding of these factors to optimise patient outcomes in a cost-effective way.

\section{Assessing Non-adherence in Glaucoma}

Adherence, as defined by the European Glaucoma Society, ${ }^{9}$ has two components: compliance and persistence. Compliance describes taking a medication as directed, with the correct dose, administration technique and dosing intervals. Persistence refers to the continuity of treatment, which may be measured by the number of prescription refills over a period of time. It is well known that adherence in glaucoma is poor, ${ }^{6}$ even in patients who are aware that their medication usage is being monitored? 
Adherence is uniformly a problem in glaucoma since the disease is asymptomatic until the very late stages, and because treatment prevents further vision loss but does not result in any perceptible improvement in visual status. Thus, there is little positive feedback to encourage adherence, and issues such as adverse effects, difficulty administering eye-drop medications and cost are also limiting factors for good adherence to treatment in most countries. A poly-drug regimen can also result in diminished effectiveness through the wash-out effect, in which multiple eye drops administered without proper spacing between them can lead to wash-out of earlier medications by later medications, before optimal ocular penetration has occurred.

The association between patient satisfaction and treatment compliance, or at least intention to or persistence in taking medications, has been documented..$^{10}$ Specifically, glaucoma patient satisfaction with treatment has been demonstrated to be a key driver of compliance. ${ }^{11}$ In a representative sample of patients treated with an IOP-lowering therapy, some associations were found between burning and stinging, vision-related quality of life and compliance. Patients with reported adverse events frequently missed instillation. ${ }^{12}$ The relative convenience and adverse effect profiles of therapies may be additional important factors in treatment decision making. More complex medication dosing schedules have been shown to negatively impact adherence in glaucoma patients. ${ }^{13-15}$

Quantifying non-adherence, and assessing the contribution of different factors, can be problematic using conventional study designs. The prevalence of self-reported non-adherence to glaucoma medication is high (27.3\% according to a Dutch survey), ${ }^{16}$ but may actually underestimate true non-adherence over time..$^{16,17}$ Randomised controlled trials can achieve strong internal validity (an unbiased estimate of difference between groups); however, their external validity (applicability to everyday clinical practice) can be limited because enrolling onto a trial may influence patient behaviour. This is particularly relevant when assessing adherence, which is likely to improve when patients know that they are under observation. Multiple obstacles to adherence have already been identified, including poor education, lack of motivation, forgetfulness, drop application and age differences. Motivation for adherence is also determined by fear of blindness and a faith in drop efficacy. ${ }^{18}$ Non-adherence may also be attributable to poor eye-drop application technique by older patients. ${ }^{19}$

We have developed a specific methodology both to assess medical outcomes over time and to identify factors that influence these outcomes..$^{20} \mathrm{An}$ initial study recruited 127 representative patients with primary open-angle glaucoma (POAG), normal-tension glaucoma or ocular hypertension via a randomly selected list of ophthalmologists. ${ }^{20}$ Disease progression, defined as a deterioration of optic nerve head and/or visual field within 2.5 years of initial diagnosis, occurred in 12 patients ( $9.5 \%$ ) during a mean follow-up of 2.4 years, but was not linked to any of the main clinical characteristics except time since diagnosis. However, there were significant correlations between disease progression and adverse effects or dissatisfaction with treatment (see Figures 1 and 2).$^{20}$ Disease progression was 3.3-fold higher in patients who experienced adverse events and 2.4-fold higher in patients who were dissatisfied with treatment. ${ }^{20}$ The study also linked treatment changes to disease progression, with an 8.7-fold increase in progression after one change (22.6\%) and a 17.7-fold increase after multiple changes (46.2\%) compared with patients who stayed on the same treatment
Figure 1: Kaplan-Meier Plots Representing Time to Optic Nerve Head and/or Visual Field Deterioration According to Adverse Effects (Wilcoxon Test) ${ }^{20}$

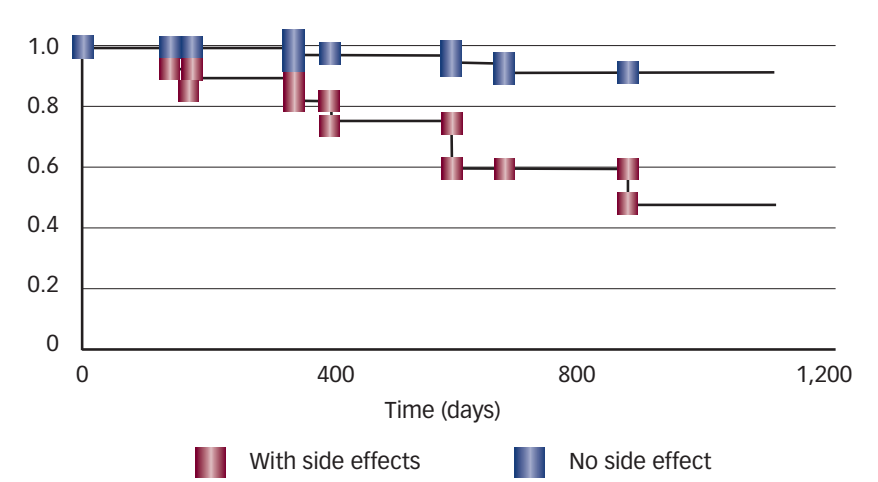

Figure 2: Kaplan-Meier Plots Corresponding to Time to Optic Nerve Head and/or Visual Field Deterioration Based on Patient Dissatisfaction with Antiglaucoma Treatment (Wilcoxon Test) ${ }^{20}$

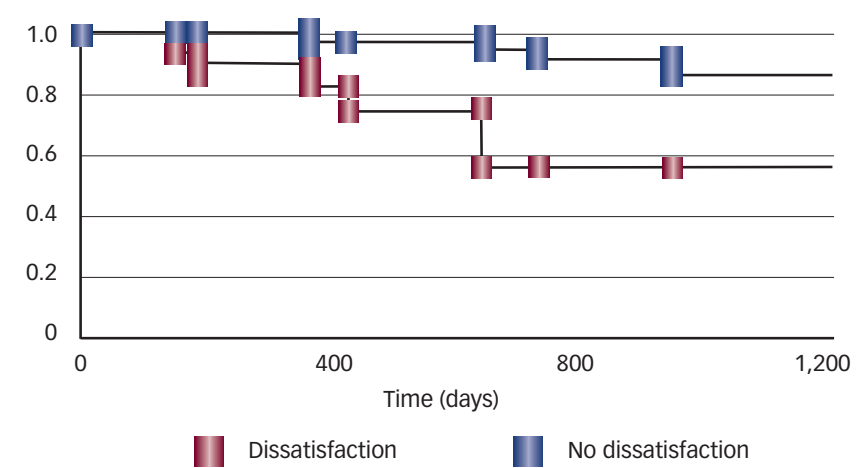

throughout the study follow-up (2.6\%).. ${ }^{20}$ Patients with disease progression appeared to have more changes in treatment and adverse events and they were also more likely to have complained about being unhappy with their treatment.

\section{Role of Treatment Adverse Effects}

Adverse effects of topical hypotensive therapy for glaucoma, such as conjunctival hyperaemia, discomfort and itching, are a leading cause of non-adherence. ${ }^{21-23}$ Patients taking fewer doses than prescribed may be at risk of having worse outcomes than those taking a higher proportion.

An analysis of 36 randomised controlled trials that included 17,511 patients identified adverse effects as the most common cause of withdrawals: $945 / 2,060$ (46\% of withdrawals) compared with $197 / 2,060$ (10\% of withdrawals) for inadequate IOP control. ${ }^{22}$ About one in 20 patients (5.4\%) withdrew overall owing to adverse effects. ${ }^{22}$ Survey results confirmed the impact of adverse effects on adherence in 'real life' as well as in clinical trials. ${ }^{12}$ Adverse effects of topical glaucoma treatment correlated with vision-related quality of life, treatment satisfaction and adherence to therapy. ${ }^{12}$

Prostaglandin analogues (PGAS) are widely used and are recommended as first-line therapy by national and international guidelines such as the European Glaucoma Society and the UK's National Institute for Health and Clinical Excellence. ${ }^{9,24}$ Studies have demonstrated that PGAs can control IOP with acceptable tolerability: patients are more likely to experience hyperaemia but less likely to have respiratory adverse 


\section{Figure 3: The Incidence of Ocular Symptoms in Different Patient Groups}

A

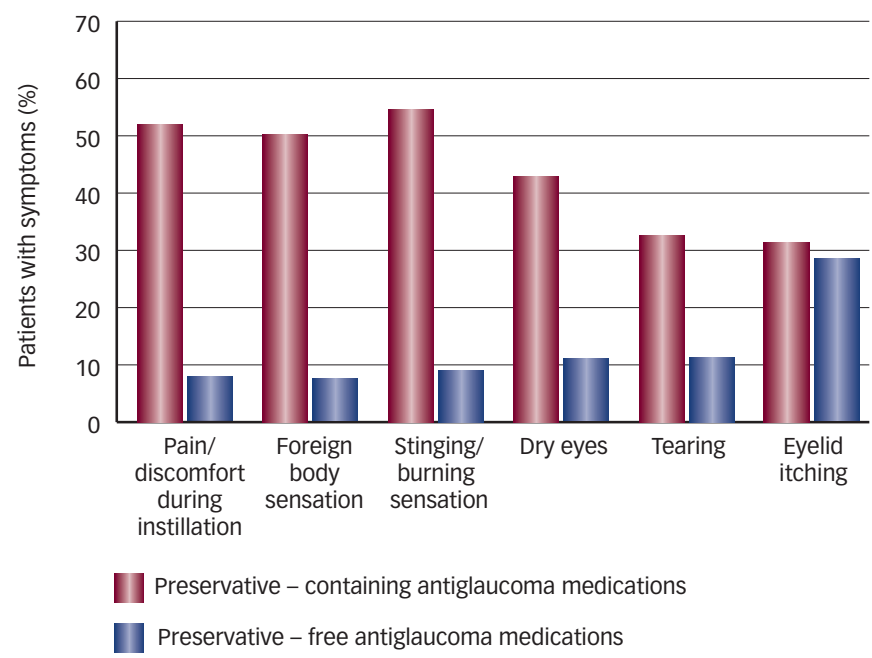

B

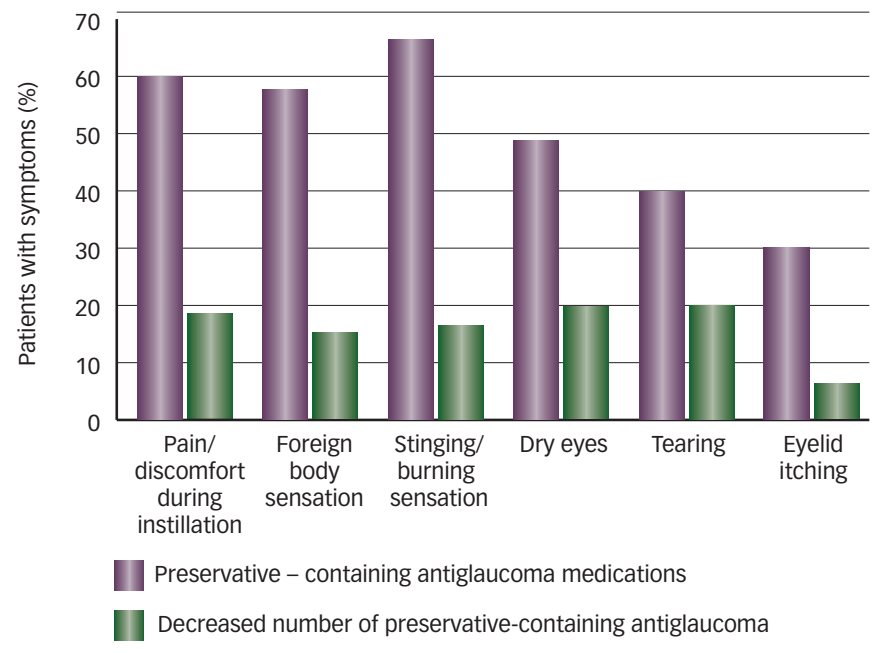

A: Patients who switched from preservative-containing to preservative-free antiglaucoma medications; B: Patients who decreased their exposure to preservative-containing antiglaucoma medications. $p<0.0001$ for all comparisons. ${ }^{34}$

effects with PGAs compared with beta-blockers. ${ }^{24}$ PGAs have a good systemic safety profile and the one-day dosing has the potential to simplify the topical regimen. Conjunctival hyperaemia is reported to be the most common adverse event in PGA-treated patients and appears to be dose-related, according to a recent randomised controlled trial comparing two doses of bimatoprost. ${ }^{25}$ Discontinuation rates were 2.2 and $6.4 \%$ in the bimatoprost 0.01 and $0.03 \%$ groups, respectively. ${ }^{25}$ Other treatment-related local adverse events include skin hyperpigmentation, eye irritation or pruritus, eyelash growth and eyelid erythema. ${ }^{25}$

Some localised adverse effects of glaucoma therapy may reflect progressive and irreversible damage, such as subconjunctival fibrosis. Long-term glaucoma treatment has been linked with cataracts, dry eye and impaired wound healing following filtration surgery. ${ }^{26,2}$ Trabeculectomy failure has been directly linked with topical glaucoma treatment and was significantly higher after more than 12 months of treatment, compared with a mean of two weeks (maximum eight weeks), of pre-operative treatment in a study of 81 patients. ${ }^{28}$ Selecting the lowest effective dose of antiglaucoma agents may be important to limit the risk of ocular damage attributable to treatment-related adverse effects. However, evidence linking eye-drop preservatives with adverse effects has fuelled debate about whether the pharmaceutically active ingredients should be our primary concern.

Benzalkonium chloride (BAK) is commonly used as a preservative in multidose bottles of topical glaucoma medications. Numerous in vitro data linked BAK with a dose-dependent toxic effect on human conjunctival and corneal epithelial cells. ${ }^{29}$ Cell survival fell with increasing BAK concentrations, whether BAK was delivered in an inert solution or combined with active glaucoma treatments, PGA and/or a beta-blocker, timolol. ${ }^{29}$ These results are consistent with previous in vitro evidence that BAK induced major irreversible damage in human conjunctival cell lines..$^{30}$ Ophthalmologists should therefore already be considering the minimum dosage necessary to achieve therapeutic benefit, but also be monitoring closely for local and systemic adverse effects, ${ }^{31}$ and the use of preservative-free formulations in subpopulations with abnormal tearing should be seriously considered. ${ }^{32}$

Some of these symptoms may actually reflect progressive and partially reversible major ocular surface changes, including subconjunctival fibrosis, increasing the risk of failure of further glaucoma surgery. All the components of the hypotensive eye drops, including the active ingredient, the $\mathrm{pH}$, the preservatives and the excipients, may be involved in the occurrence of these adverse effects. It is thus important to identify the agents involved and the mechanisms of these adverse effects in order to select a therapy minimising their risk and the discomfort felt by patients, thereby increasing the likelihood of good compliance. Preservative-free solutions should be considered when available. ${ }^{33,34}$

Clinical research has also shown that adverse effects are significantly less common with preservative-free preparations compared with standard preservative-containing eye drops. ${ }^{35,36}$ Large-scale epidemiological studies have reported higher frequencies of all recorded symptoms and signs of irritation (palpebral, conjunctival and corneal) in patients using preservative-containing versus preservative-free preparations. ${ }^{35,36}$ A cross-sectional study including 9,658 patients in four European countries found that all symptoms were more than twice as common in the group using preservative-containing treatments: 48 versus $19 \%$ had discomfort or pain during instillation; 48 versus $20 \%$ reported stinging or burning; 42 versus $15 \%$ experienced foreign body sensation; and 35 versus $16 \%$ had dry eye sensation in preservative-exposed versus preservative-free groups, respectively. ${ }^{35} \mathrm{~A}$ similar cross-sectional study in 4,107 patients revealed similar results, for example discomfort upon instillation in 43 versus $17 \%{ }^{36}$ Both studies also showed that eliminating or reducing preservative exposure dramatically reduced symptom frequency (see Figure 3). ${ }^{35,36}$

\section{Cost-effectiveness}

A number of groups have attempted to map cost-effectiveness in a variety of glaucoma populations. As would be expected, the resource consumption associated with this chronic disease demonstrates greater medical effort as disease severity worsens. Increased medical effort is prompted by physicians in order to slow progression of the condition, but it is also driven by patient concerns. Glaucoma therapy is cost-effective compared with no treatment; however, simulation models are inconsistent regarding when to treat ocular hypertension and comparing different therapeutic interventions. ${ }^{37}$ However, health policy involves the investment of public resources, 
and cost-effectiveness analyses for POAG screening may be weighted by the degree of uncertainty regarding whether glaucoma screening can be effectively and reliably achieved.

Glaucoma treatment should aim to maintain visual function and vision-related quality of life at a sustainable cost (including adverse effects and inconvenience, as well as financial costs), according to European guidelines. ${ }^{9}$ A growing range of treatment options, combined with finite resources, means that cost-effectiveness is an increasingly important consideration. There has been an historical shortage of high-quality health economics data in glaucoma, but several studies have aimed to address this lack in recent years. ${ }^{938-45}$

\section{Intraocular Pressure-lowering Medications}

A study of long-term resource use in 194 patients with glaucoma in four European countries (France, Germany, Italy and the UK) revealed a significant linear increase in direct ophthalmology costs with worsening disease severity, estimated at $€ 455 /$ person-year at disease stage 0 versus €969/person-year at disease stage 4 (see Figure 4). ${ }^{45}$ The number of glaucoma medications (at least $42 \%$ of direct costs, assuming full adherence) increased linearly from disease stage 0 to $5 .{ }^{45}$ There was a linear rise in the number of ophthalmologist visits per person-year as disease stage worsened. ${ }^{45}$ Survey data have also shown that patients may visit their ophthalmologist more often if they are dissatisfied with treatment, with 3.5 visits/year versus two. ${ }^{27}$ visits/year for dissatisfied versus satisfied patients. ${ }^{12}$ Traverso et al. concluded that effective disease control was the most cost-effective approach, with potential cost savings of 30-50 \% for preventing progression from disease stage 0 or 1 to stage 4 or $5 . .^{45}$

More recent studies have identified treatment changes as a major contributor to rising costs. ${ }^{42,43}$ Danish registry data on 27,380 patients who began antiglaucoma therapy between 2002 and 2007 revealed that, after a mean 2.4-year follow-up, only $37 \%$ were still using their original treatment regimen. ${ }^{42}$ A further $21 \%$ had switched therapy once, while $43 \%$ had experienced three or more treatment changes. ${ }^{42}$ Medication accounted for $61 \%$ of initial direct healthcare costs in this study, but fell to $51 \%$ after three treatment changes while other costs increased. ${ }^{42}$ Annual costs of ophthalmologist visits, for example, increased from an initial €110 to €239 in patients on their third or more treatment regimen. ${ }^{42}$ Annual healthcare costs per patient were $€ 305$ during their first treatment regimen, rising to $€ 744$ after three treatment changes. ${ }^{42}$

Another recent publication reported rising costs with medication changes, and concluded that effective and well-tolerated initial treatment was the most cost-effective approach to glaucoma management. ${ }^{43}$ A 10-year Markov model based on the UK health system and populated with data from 93 studies revealed only small differences in rates of failure to control IOP for first-line monotherapy using different PGAs. ${ }^{43}$ However, reduced hyperaemia led to prolonged treatment in one group and 10-year cumulative costs were lowest despite higher drug acquisition costs versus the alternative PGAs. ${ }^{43}$ Drug costs accounted for just 8-13\% of total costs of glaucoma in this model, which included costs of outpatient visits, surgery and indirect costs of low vision, such as care for a blind or partially sighted patient. ${ }^{43}$

A novel analysis of data for 1,816 patients, taken from the UK General Practitioner Research Database, also concluded that the most durable treatment sequence was the most cost-effective. ${ }^{40}$ This analysis linked
Figure 4: Direct Cost of Glaucoma Treatment in Europe per Person-year by Stage ${ }^{45}$

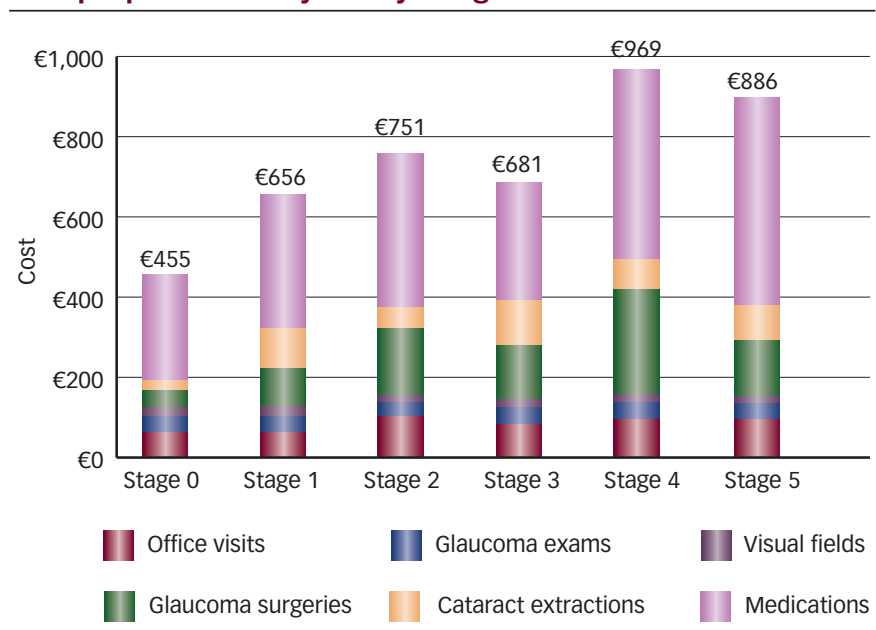

the most persistent treatment sequence with lower monthly costs of drugs, consultations and total monthly costs compared with alternative treatment sequences. ${ }^{40}$ The most cost-effective treatment sequence started with a different first-line PGA to the above study, but the results were consistent in that they linked treatment persistence with cost savings. ${ }^{40,43}$

The first prospective study of long-term costs and outcomes of PGA treatment in clinical practice reported a four-year follow-up of 467 patients with POAG or ocular hypertension who were starting first- or second-line PGA treatment for insufficiently controlled IOP. ${ }^{39}$ The results linked PGA therapy with very little progression over four years, and $52 \%$ of patients remained on their initial treatment throughout the study. ${ }^{39}$ There was no overall increase in treatment costs despite the relatively high acquisition costs of PGAs. The authors estimated that the costs of surgery and consultations had fallen by around 70 and $34 \%$, respectively, with increased use of PGAs. Both baseline IOP and treatment switches were linked to increased healthcare costs..$^{39}$ First-line PGA therapy appeared to be significantly more effective than initial treatment with a beta-blocker, providing more days of IOP control primarily owing to the longer time until initial treatment failure..$^{46}$

There is also evidence that, while screening of the general population may not be cost-effective, screening of at-risk groups including the elderly and those with a family history of glaucoma may be cost-effective. ${ }^{38}$ Since models estimate that the lifetime cost of ocular hypertension is higher than that of glaucoma, with the social costs of each disease not being compared, first-line use of the most effective therapy would reduce medical and social costs. ${ }^{41}$

\section{Cost Implications of Laser Trabeculoplasty}

A case-control analysis of 1,145 patients with POAG who underwent laser trabeculoplasty (LTP) versus 2,290 matched controls linked this procedure with increased total healthcare and ophthalmology-related charges during the following 12 months. ${ }^{44}$ Non-pharmacy charges increased by US $\$ 4,641$ following LTP compared with a US $\$ 1,293$ increase in control patients, and ophthalmology-related charges increased by US $\$ 1,364$ following LTP compared with just US $\$ 30$ in controls. ${ }^{44}$ Twice as many patients underwent cataract surgery in the 12 months after LTP compared with the previous 12 months (50 [4.37\%] versus 24 [2.10]), compared with no increase in the control group. ${ }^{44}$ Repeat LTP appeared to be a major driver of increased costs following LTP: 507 
Figure 5: Self-reported Glaucoma Education and Adherence According to Health Literacy (\%)

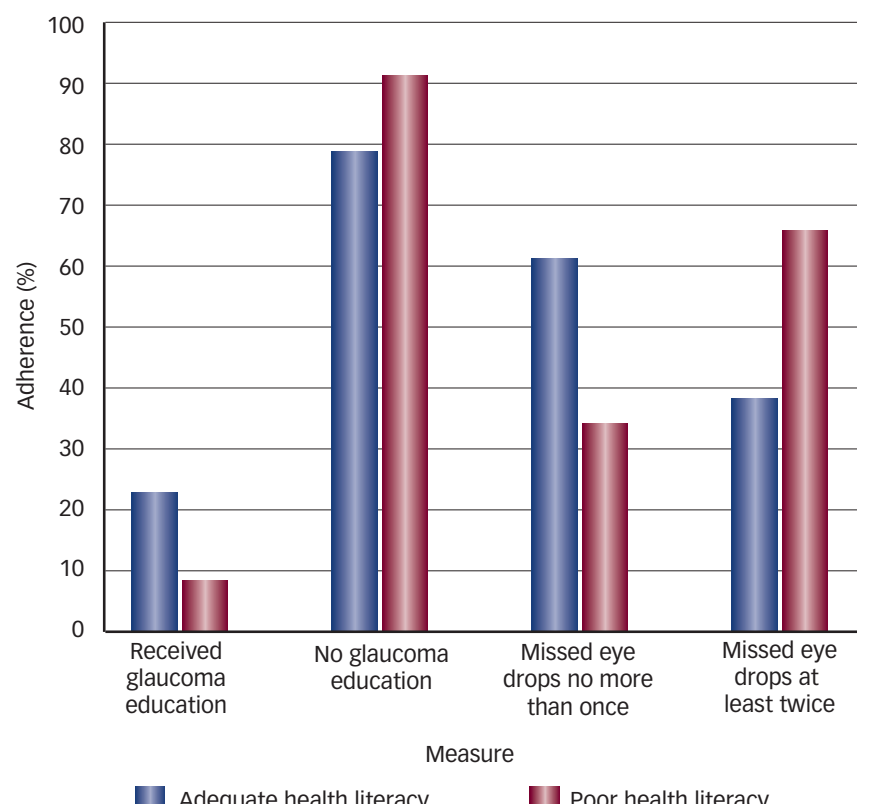

Data taken from Juzych et al., 2008. ${ }^{49}$

patients (44 \%) required repeat LTP during the following 12 months. ${ }^{44}$ There was a US\$2,101 increase in ophthalmology-related non-pharmacy charges for patients who had a repeat LTP compared with US\$773 in patients who did not require repeat LTP within one year after index LTP. ${ }^{44}$ A Canadian study has also shown that selective LTP as primary therapy, at a per-patient level, offers a modest potential cost saving over primary medical therapy in the management of POAG. ${ }^{47}$

\section{Patient Management}

The efficacy and cost-effectiveness of medical treatment rely on adequate adherence, but chronic adherence rates are likely to be poorer than either randomised controlled trial or survey data suggest. It is probably best to assume low adherence in the clinic and apply management strategies that can optimise adherence. For example, selecting preservative-free medications may avoid poor adherence attributable to preservative-related adverse effects.

The physician's role goes beyond treatment selection and patient monitoring, however; education and counselling are particularly important to support patients with chronic conditions such as glaucoma. A cross-sectional study in 408 subjects highlighted the scope for improvement: misconceptions about the disease and its treatment were common in both newly diagnosed and established glaucoma patients. ${ }^{48}$ For example, $36 \%$ of established glaucoma patients believed that watery eyes indicated increased pressure inside the eye, $80 \%$ were unaware that topical glaucoma treatments could have systemic adverse effects and $48 \%$ thought that they would have symptoms warning them of disease progression. ${ }^{48}$ Knowledge scores were highest for patients who had been referred to an optometrist, shared the same first language as their healthcare provider or received private treatment, which are all factors that could influence their health information and counselling. ${ }^{48}$

Other evidence has linked poor disease understanding (or health literacy) with impaired treatment adherence and patient outcomes. ${ }^{49,50}$
Health literacy (Rapid Assessment of Adult Literacy in Medicine [REALM] score) was the only factor that predicted the number of medication refills requested over six months in a study of 197 patients with glaucoma. ${ }^{50}$ There was no link between refill rates and age, gender, race or level of education in this study..$^{50} \mathrm{~A}$ cross-sectional study of 204 patients with established glaucoma also linked poor health literacy with both a lack of glaucoma education and worsened glaucoma outcomes (see Figure 5) 4. $^{4}$ Patients with poor health literacy had higher rates of missed appointments and missed eye drops (see Figure 5$)^{49}$ Glaucoma worsening was also increased in patients without glaucoma education. ${ }^{49}$

There is evidence that supplementary education for physicians and patients can improve patients' disease understanding..$^{51,52}$ In one study, physicians asked over twice as many questions about adherence per visit (10.1 versus 4.1), and more than four times as many visits included questions about adherence after training compared with before training (82 versus $18 \%$ ). ${ }^{52}$ While combined fixed therapies and preservative-free topical treatments should also improve adherence and quality of life by simplifying instillation and tolerance of eye drops, physicians should give clear and precise information about the expected benefits of the therapy, the disease and its risk of progression..$^{53}$

Another study has demonstrated that formal education sessions can significantly improve patients' understanding of glaucoma: the mean number of correctly answered glaucoma questions increased significantly and was sustained at 10 months of follow-up (3.81 correct answers pre-session versus 5.2610 months after the session)..$^{51}$

Useful guidance has been provided, including optimal use of evidence-based clinical guidelines for treatment and keeping the therapy plan straightforward and tailored to patient needs and preferences. It is important to recognise early in the treatment plan the possible obstacles to compliance and persistence and plan interventions accordingly. Progression of disease and quality of life should be monitored and timely additional interventions instituted as needed. ${ }^{54}$

Comprehension of the disease generates commitment, which in turn produces adherence to treatment. It is thus critical to address patients' concerns and beliefs about glaucoma and ocular hypotensive therapies. Well-tolerated medical treatments should be used, as the higher the frequency of adverse effects, the greater the number of missed instillations. The amount of instillations per day should be reduced, as the more complex the regimen, the higher the frequency of missed instillations. ${ }^{8}$

\section{Discussion}

The management of glaucoma remains a clinical challenge. Treatment is currently limited to risk factor changes in the form of reduction of IOP. IOP lowering has been shown to be effective in reducing the risk of both the development and progression of glaucoma, at all the stages of the disease. There remains significant unmet need in the glaucoma therapeutic arena. Tolerability is crucial for sustained adherence and should be a prime consideration when prescribing IOP-lowering medication. Choosing a preservative-free formulation is a relatively simple way to reduce some adverse effects such as hyperaemia. As more data come to light underscoring the potential detrimental effects of chronic BAK exposure to the ocular surface, the role of preservative-free IOP-lowering formulations continues to expand. Preservative-free formulations of several antiglaucoma drugs are also available. 
Effective glaucoma management calls for a holistic approach to optimise lifelong outcomes. Treatment selection should take account of long-term effectiveness, acknowledging that the initially most cost-effective choice may not prove to be the most effective over time. Prolonged efficacy demands sustained adherence, but achieving good adherence means overcoming barriers such as adverse effects and poor disease understanding.

Practical strategies to maximise adherence include selecting a simple regimen with clear instructions, and physicians should ensure that patients understand their disease and its treatment sufficiently to encourage good adherence.

\section{Future Developments}

Several novel medication delivery systems are in development with the goal of improving adherence as well as improving pharmacokinetics by delivering a continuous small dose. For example, a surgically implanted programmable mini-pump offers the potential for long-term drug delivery directly inside the eye. ${ }^{55,56}$ This technology could minimise non-adherence by removing the need for daily administration (refills could last for several months) while reducing systemic exposure and allowing accurate tailored dosing. ${ }^{55}$ considerable efforts are also being put into creating intelligent devices that could potentially sense when and how much dose is required and then automatically release it from reservoirs. ${ }^{57}$ Researchers are also exploring the potential for glaucoma drug delivery using injectable microspheres, which could replace daily self-administration with an injection every few months. ${ }^{58-60}$

Another option under investigation is to deliver IOP-lowering drugs via hydrogel contact lenses. ${ }^{61-64}$ Preliminary evidence suggests that this is a feasible method of antiglaucoma drug delivery. ${ }^{63}$ These novel technologies are designed to improve clinical efficacy by providing many delivery alternatives and can manage IOP for several months.
Another crucial unmet need in glaucoma management is the development of therapies that work independently of IOP reduction, namely by neuroprotection or neurodegeneration. Glaucoma is a multifactorial optic neuropathy, and elevated IOP is only one cause of optic nerve damage. Many glaucoma patients lose vision despite an apparently normal IOP and there is no specific IOL level below which one is safe from glaucoma. Neurotropic factors have shown ocular neuroprotective activity in animal models and could prove valuable for patients whose glaucoma continues to progress despite IOP reduction. ${ }^{65-67}$ However, conventional oral or eye-drop formulations are unsuitable for these agents, so their neuroprotective potential depends on the development of specialised delivery methods that can supply the medications in a continuous, restricted manner to the retina and optic nerve. Medication delivery systems may improve efficacy, decrease adverse effects, increase patient adherence and, ultimately, maintain vision for glaucoma patients.

Insufficient compliance may lead to disease progression through under-treatment and also by introducing IOP variability throughout the day. Short-term and long-term IOP fluctuations may be a significant risk factor for disease progression. The ability to deliver IOP-lowering therapy at a steady state over periods longer than 24 hours is needed, and may directly improve compliance. The ideal solution would be a sustained-release delivery device that would offer steady-state pharmacokinetics and obviate daily adherence. Some subconjunctivally or intravitreally injected implants, loaded with antiglaucoma drugs, are currently under development.

\section{Conclusion}

Public health decisions in glaucoma therapy should take a broad economic view incorporating the lifetime duration of the disease. There remains a position within and outside the healthcare system for therapeutic innovations with important economic consequences that bring high added value to patients."
1. Alemu AM, Kristoffersen CJ, Kristoffersen MS, et al., Long-term benefit of reduced intraocular pressure in primary open-angle glaucoma patients in Ethiopia, Eur J Ophthalmol, 2010;20:310-5

2. Kass MA, Heuer DK, Higginbotham EJ, et al., The Ocula Hypertension Treatment Study: a randomized trial determines that topical ocular hypotensive medication delays or prevents the onset of primary open-angle glaucoma, Arch Ophthalmol, 2002;120:701-13; discussion 829-30.

3. Weinreb RN, Khaw PT, Primary open-angle glaucoma, Lancet, 2004;363:1711-20

4. Nordmann JP, Baudouin C, Renard JP, et al., Identification of noncompliant glaucoma patients using Bayesian networks and the Eye-Drop Satisfaction Questionnaire, Clin Ophthalmol, 2010:4:1489-96

5. Patel SC, Spaeth $\mathrm{GL}$, Compliance in patients prescribed eyedrops for glaucoma, Ophthalmic Surg, 1995;26:233-6.

eyedrops for glaucoma, Ophthalmic Surg, $1995,26.233-6$.
6. Winfield AJ, Jessiman D, Williams A, et al., A study of the causes of non-compliance by patients prescribed eyedrops, Br J Ophthalmol, 1990;74:477-80.

7. Tsai JC, A comprehensive perspective on patient adherence to topical glaucoma therapy, Ophthalmology, 2009;116:S30-6.

8. Fogagnolo P, Rossetti L, Medical treatment of glaucoma: present and future, Expert Opin Investig Drugs, 2011;20:947-59.

9. European Glaucoma Society, Terminology and Guidelines for Glaucoma, 3rd ed., Savona, Italy: Dogma, 2008;25-31.

10. Woodside AG, Frey LL, Daly RT, Linking service quality, customer satisfaction, and behavioral intention, J Health Care Mark, 1989;9:5-17.

11. Day DG, Sharpe ED, Atkinson MJ, et al., The clinical validity of the treatment satisfaction survey for intraocular pressure in ocular hypertensive and glaucoma patients, Eye (Lond), 2006;20:583-90.

12. Nordmann JP, Auzanneau N, Ricard S, et al., Vision related quality of life and topical glaucoma treatment side effects, Health Qual Life Outcomes, 2003;1:75

13. Gurwitz JH, Glynn RJ, Monane M, et al., Treatment for glaucoma: adherence by the elderly, Am J Public Health 1993:83:711-6.

14. Olthoff CM, Schouten JS, van de Borne BW, et al.,
Noncompliance with ocular hypotensive treatment in patients with glaucoma or ocular hypertension an evidencebased review, Ophthalmology, 2005;112:953-61.

15. Robin AL, Novack GD, Covert DW, et al., Adherence in glaucoma: objective measurements of once-daily and adjunctive medication use, Am J Ophthalmol, 2007;144:533-40.

16. Olthoff CM, Hoevenaars JG, van den Borne BW, et al., Prevalence and determinants of non-adherence to topica hypotensive treatment in Dutch glaucoma patients, Graefes Arch Clin Exp Ophthalmol, 2009;247:235-43.

17. Jerant A, DiMatteo R, Arnsten J, et al., Self-report adherence measures in chronic illness: retest reliability and predictive validity, Med Care, 2008:46:1134-9.

18. Lacey J, Cate H, Broadway DC, Barriers to adherence with glaucoma medications: a qualitative research study, Eye (Lond), 2009;23:924-32.

19. Aptel F, Masset H, Burillon $C$, et al., The influence of disease severity on quality of eye-drop administration in patients with glaucoma or ocular hypertension, Br J Ophthalmol, 2009;93:700-1.

20. Denis P, Lafuma A, Berdeaux G, Medical outcomes of glaucoma therapy from a nationwide representative survey, Clin Drug Investig, 2004;24:343-52.

21. Alm A, Grunden JW, Kwok KK, Five-year, multicenter safety study of fixed-combination latanoprost/timolol (Xalacom) for open-angle glaucoma and ocular hypertension, J Glaucoma 2011:20:215-22.

22. Stewart WC, Demos CM, Turner MK, et al., Risk factors for subject withdrawals in clinical trials evaluating glaucoma medications, Graefes Arch Clin Exp Ophthalmol, 2010;248:1007-12

23. Zimmerman T, Hahn SR, Gelb L, et al., The effect of hyperaemia on open-angle glaucoma (OAG) treatment, Annual Meeting of the European Society of Ophthalmology (SOE), 9-12 June, Vienna, Austria 2007: abstract FP-GLA-036.

24. National Collaborating Centre for Acute Care, Glaucoma: Diagnosis and management of chronic open angle glaucom and ocular hypertension. Methods, evidence and guidance, London: NICE; 2009. Available at: http://guidance.nice.org.uk/ nicemedia/live/12145/43887/43887.pdf (accessed 26 September 2011).

25. Katz $\mathrm{L}$, Cohen JS, Batoosingh AL, et al., Twelve-month, randomized, controlled trial of bimatoprost $0.01 \%, 0.0125 \%$, and $0.03 \%$ in patients with glaucoma or ocular hypertension, Am J Ophthalmol, 2010:149:661-71 e1.

26. Broadway DC, Grierson I, O'Brien C, et al., Adverse effects of topical antiglaucoma medication. II. The outcome of filtration surgery, Arch Ophthalmol, 1994;112:1446-54.

27. Franks WA, Renard JP, Cunliffe IA, et al., A 6-week, doublemasked, parallel-group study of the efficacy and safety of travoprost $0.004 \%$ compared with latanoprost 0:005\%/timolo $0.5 \%$ in patients with primary open-angle glaucoma or ocular hypertension, Clin Ther, 2006;28:332-9.

28. Lavin MJ, Wormald RP, Migdal CS, et al., The influence of prior therapy on the success of trabeculectomy, Arch Ophthalmol, 1990;108:1543-8.

29. Ammar DA, Noecker RJ, Kahook MY, Effects of benzalkonium chloride- and polyquad-preserved combination glaucoma medications on cultured human ocular surface cells, Adv Ther 2011;28:501-10.

30. De Saint Jean M, Debbasch C, Brignole F, et al., Toxicity of preserved and unpreserved antiglaucoma topical drugs in an in vitro model of conjunctival cells, Curr Eye Res, 2000;20:85-94.

31. Servat JJ, Bernardino CR, Effects of common topica antiglaucoma medications on the ocular surface, eyelids and periorbital tissue, Drugs Aging, 2011;28:267-82.

32. Tressler CS, Beatty R, Lemp MA, Preservative use in topical glaucoma medications, Ocul Surf, 2011;9:140-58.

33. Aptel F, Denis P, Baudouin C, [Managing treatment side effects: the respective roles of the active ingredient and the preservative], J Fr Ophtalmol, 2011;34:409-12. Article in French.

34. Baudouin C, Detrimental effect of preservatives in eyedrops: implications for the treatment of glaucoma, Acta Ophthalmol. 2008;86:716-26.

35. Jaenen N, Baudouin C, Pouliquen P, et al., Ocular symptoms and signs with preserved and preservative-free glaucoma medications, Eur J Ophthalmol, 2007;17:341-9. 
36. Pisella PJ, Pouliquen P, Baudouin C, Prevalence of ocular symptoms and signs with preserved and preservative free glaucoma medication, Br J Ophthalmol, 2002;86:418-23.

37. Tuulonen A, Cost-effectiveness of screening for open angle glaucoma in developed countries, Indian I Ophthalmol, 2011:59(Suppl.):S24-30.

38. Hirneiss $C$, Niedermaier $A$, Kernt $M$, et al., [Health-economic aspects of glaucoma screening], Ophthalmologe,

2010;107:143-9. Article in German.

39. Kobelt G, Texier-Richard B, Buchholz P, et al., Treatment of glaucoma in clinical practice: four-year results from a patient registry in France, I Glaucoma, 2010;19:199-206.

40. Lafuma A, Salmon JF, Robert J, et al., Treatment persistence and cost-effectiveness of latanoprost/latanoprost-timolol bimatoprost/bimatoprost-timolol, and travoprost/travoprosttimolol in glaucoma: an analysis based on the United Kingdom general practitioner research database, Kingdom general practitione
Clin Ophthalmol, 2011;5:361-7.

41. Nordmann JP, Lafuma A, Berdeaux G, Modelling the lifetime economic consequences of glaucoma in France, J Med Econ, 2009;12:9-16.

42. Olsen J, Berdeaux G, Skov J, Glaucoma costs in Denmark in treatment naive patients, Acta Ophthalmol.

2011; (Epub ahead of print).

43. Orme M, Collins S, Loftus J, Long-term medical management of primary open-angle glaucoma and ocular hypertension in the UK: optimizing cost-effectiveness and clinic resources by minimizing therapy switches, J Glaucoma,

2011; (Epub ahead of print).

44. Pasquale LR, Walt JG, Stern LS, et al., Healthcare charges in patients with glaucoma who undergo laser trabeculoplasty, Adv Ther, 2009;26:1084-96.

45. Traverso CE, Walt JG, Kelly SP, et al., Direct costs of glaucoma and severity of the disease: a multinational long term study of resource utilisation in Europe, Br 1 Ophthalmol,
2005;89:1245-9.

46. Rouland JF, Le Pen C, Naturalistic, prospective study of glaucoma and ocular hypertension treatment in France: strategies, clinical outcomes, and costs at 1 year, Eur I Ophthalmol, 2003:13(Suppl. 4):S5-20.

47. Lee R, Hutnik CM, Projected cost comparison of selective laser trabeculoplasty versus glaucoma medication in the Ontario Health Insurance Plan, Can J Ophthalmol, 2006;41:449-56.

48. Danesh-Meyer HV, Deva NC, Slight C, et al., What do people with glaucoma know about their condition? A comparative cross-sectional incidence and prevalence survey, Clin Experiment Ophthalmol, 2008;36:13-8.

49. Juzych MS, Randhawa S, Shukairy A, et al., Functional health literacy in patients with glaucoma in urban settings, Arch Ophthalmol, 2008:126:718-24.

50. Muir KW, Santiago-Turla C, Stinnett SS, et al., Health literacy and adherence to glaucoma therapy, Am I Ophthalmol, 2006;142:223-6.

51. Blondeau $P$, Esper $P$, Mazerolle $E, A n$ information sessio for glaucoma patients, Can J Ophthalmol, 2007;42:816-20.

52. Hahn SR, Friedman DS, Quigley HA, et al., Effect of patient-centered communication training on discussion and detection of nonadherence in glaucoma, Ophthalmology, 2010:117:1339-47 e6.

53. Nordmann JP, Akesbi J, [Improve adherence in glaucoma patients: a doctor's duty], J Fr ophtalmol 2011:34:403-8. Article in French.

54. Kulkarni SV, Damji KF, Buys YM, Medical management of primary open-angle glaucoma: best practices associated with enhanced patient compliance and persistency, Patient Prefer Adherence, 2008;2:303-14.

55. Saati S, Lo R, Li PY, et al., Mini drug pump for ophthalmic use, Trans Am Ophthalmol Soc, 2009;107:60-70.

56. Saati S, LO R, Li PY, et al., Mini drug pump for ophthalmic use, Curr Eye Res, 2010;35:192-201

57. Staples M, Daniel K, Cima MJ, et al., Application of micro- and nano-electromechanical devices to drug delivery, Pharm Res, 2006;23:847-63.

58. Bao W, Zhou J, Luo J, et al., PLGA microspheres with high drug loading and high encapsulation efficiency prepared by a novel solvent evaporation technique, J Microencapsul, 2006;23:471-9.

59. Bertram JP, Saluja SS, MCKain J, et al., Sustained delivery of timolol maleate from poly(lactic-co-glycolic acid)/poly(lactic acid) microspheres for over 3 months, J Microencapsul, 2009;26:18-26.

60. Fu K, Harrell R, Zinski K, et al., A potential approach for decreasing the burst effect of protein from PLGA microspheres, J Pharm Sci, 2003:92:1582-91.

61. Hiratani $\mathrm{H}$, Alvarez-Lorenzo $\mathrm{C}$, Timolol uptake and release by imprinted soft contact lenses made of $\mathrm{N}, \mathrm{N}$-diethylacrylamide and methacrylic acid, I Control Release, 2002;83:223-30.

62. Hoffman AS, Hydrogels for biomedical applications, Adv Drug Deliv Rev, 2002;54:3-12.

63. Schultz CL, Poling TR, Mint JO, A medical device/drug delivery system for treatment of glaucoma, Clin Exp Optom, 2009;92:343-8.

64. White CJ, Byrne ME, Molecularly imprinted therapeutic contact lenses, Expert Opin Drug Deliv, 2010;7:765-80.

65. Grozdanic SD, Lazic T, Kuehn MH, et al., Exogenous modulation of intrinsic optic nerve neuroprotective activity, Graefes Arch Clin Exp Ophthalmol, 2010;248:1105-16.

66. Kyhn MV, Klassen H, Johansson UE, et al., Delayed administration of glial cell line-derived neurotrophic factor (GDNF) protects retinal ganglion cells in a pig model of acute retinal ischemia, Exp Eye Res, 2009;89:1012-20.

67. Ward MS, Khoobehi A, Lavik EB, et al., Neuroprotection of retinal ganglion cells in DBA/2 I mice with GDNF-loaded biodegradable microspheres, I Pharm SCi, 2007:96:558-68. 